\title{
APPLICATION OF PID ALGORITHM IN DRIVE FAULT-TOLERANT CONTROL OF FOUR-WHEEL DRIVE ELECTRIC VEHICLE
}

\author{
Jing Wang ${ }^{1}$ \\ ${ }^{1}$ Puyang Vocational and Technical College, Henan 457000, China. \\ Email: wangjinghenan2001@163.com
}

\begin{abstract}
Objective: The objective of this study is to study the application of PID algorithm in the drive fault-tolerant control of four-wheel drive electric vehicle, and to improve the stability of electric vehicle. Method: The dynamic model of vehicle is analysed and modelled to obtain the reference value of vehicle running in steady state. Then, the hub motor is used to study the direct torque of a single Brushless Direct Current Motor (BLDCM). The fuzzy PID controller based on the yaw rate and the sideslip angle of the centre of mass is designed. In the environment of Simulink and CarSim, the whole vehicle simulation model of electric vehicle is established. The whole vehicle system is simulated and analysed. Results: Under the condition of straight line, lane change and overtaking, the running state of the fault motor in different time, different location and different location is simulated. The simulation results show the validity and correctness of the design strategy. Conclusion: In this study, the designed drive fault-tolerant control strategy can effectively control the running stability of the vehicle in different states, so that the vehicle can still run stably in case of hub motor failure, and improve the safety of drivers and passengers.
\end{abstract}

Keywords: Four-Wheel Drive; Drive Fault-Tolerant Control; PID Algorithm; Electric Vehicle.

\section{Introduction}

In response to the concept of green travel and green development advocated by the state, new energy vehicles have gradually become an unstoppable trend in the development of the automobile industry. As the leader of new energy vehicles, the power of pure electric vehicles can be completely provided by renewable resources - electricity. According to different driving structures, pure electric vehicles can be divided into two ways: central motor drive and electric wheel drive [1]. In essence, the pure electric vehicle driven by the central motor only uses the motor to replace the traditional internal combustion engine. There are still clutch, gearbox, differential and other structures in the layout of the whole vehicle.

Compared with the traditional automobile machinery, the pure electric vehicle driven by the electric wheel has undergone tremendous changes in structure, abandoning the traditional mechanical differential. The function of the mechanical differential is realized by using the electronic differential between wheels, which greatly simplifies the structure of the car $[2,3]$. As a typical driving mode of electric wheel, hub motor drive integrates power, transmission and braking devices into the hub, which makes the driving efficiency of electric vehicle get a qualitative leap. Therefore, the hub motor drive mode has gradually become a hot research direction in the field of electric vehicles [4].

Fault tolerant control is first proposed by Swedish professor Astrom. Its main function is to keep stable operation when a sensor or actuator in a closed-loop control system fails, and to meet certain performance requirements. Some scholars have designed the distribution control strategy of the braking / driving force of four-wheel drive vehicles by using the model prediction algorithm for the stability of the distributed drive electric vehicles during the limit working condition, and designed the self-tuning fault-tolerant control algorithm for the operation safety of the vehicles after actuator failure [5]. Through the self-developed experimental platform, it is verified that the designed torque distribution strategy improves the yaw stability of the distributed drive vehicle under the limit condition, and the fault-tolerant control algorithm ensures the safety of the vehicle during operation when the actuator fails [6]. Based on the design of electronic differential, some scholars designed a four-wheel independent drive vehicle to improve the driving stability of the vehicle. The upper layer is a sliding mode controller, whose function is to calculate the longitudinal and transverse forces of the vehicle in an ideal state. The lower layer is torque controller, which controls the driving force of each wheel $[7,8]$. 
On the basis of the above research status, the drive fault-tolerant control strategy of four-wheel drive electric vehicle is further explored in this study. Firstly, the vehicle dynamic model is analysed and modelled to obtain the reference value of the vehicle running in the steady state. Then, the direct torque of a single Brushless Direct Current Motor (BLDCM) is studied. The fuzzy PID controller based on yaw rate and centroid sideslip angle is designed to control the output of the optimal additional yaw moment, optimize the distribution of four-wheel torque, reduce yaw rate and centroid sideslip angle, so as to improve the stability of the vehicle. In the environment of Simulink and CarSim, the whole vehicle simulation model of electric vehicle is established to simulate and analyse the whole vehicle system.

\section{Methods}

\subsection{Dynamics of Four-wheel drive vehicle}

The four-wheel drive electric vehicle is a complex body composed of many systems, and the force situation in its operation process is very complex.

Therefore, the establishment of a dynamic model which can accurately reflect the vehicle's motion performance is the basis of the research on the drive fault-tolerant control of the four-wheel drive electric vehicle. Vehicle is a multi-degree of freedom and nonlinear strong coupling object, so the vehicle dynamics model is simplified without affecting the purpose of this study. A 7-degree of freedom (DOF) vehicle model and a tire model are established to analyse the vehicle dynamics, and a linear 2-DOF dynamic model is constructed as the reference model of vehicle stability and steering characteristics, which paves the way for the subsequent research of fault-tolerant control strategy.

The four hub motors of the hub driven electric vehicle can be controlled independently.

Therefore, in order to maintain the stability of the vehicle in the running process, it is necessary to design the corresponding algorithm to control the torque output of each hub motor, so as to fully coordinate the torque distribution of the vehicle in different driving conditions. When one or two hub motors fail to repair during the operation of the vehicle, and the torque required for the operation of the vehicle cannot be output, if the corresponding measures are not taken, it will lead to the incalculable danger of the vehicle. Therefore, it is necessary to design the corresponding drive faulttolerant control strategy when the hub motor fails, so that the missing drive torque of the failed motor can be compensated by the control strategy, so as to ensure the stability and safety of the vehicle when the hub motor fails.

It is assumed that the vertical, roll and pitch motions have the same influence on the vehicle body; the difference changes of each tire itself are ignored, and the mechanical characteristics are assumed to be the same; the angle of two wheels is assumed to have no difference changes, and the transmission is proportional; the effects of torsional vibration, shimmy and other vibrations are ignored.

Then, a 7-DOF vehicle model is established. The kinematic model of the whole vehicle is shown in Figure 1.

Equation of longitudinal motion along the $\mathrm{x}$-axis (equation 1):

$$
\mathrm{ma}_{x}=F_{x f l} \cos \delta_{l}+F_{x f r} \cos \delta_{r}-F_{y f l} \sin \delta_{l}-F_{y f r} \sin \delta_{r}+F_{x r l}+F_{x r r}
$$

Equation of longitudinal motion along the y-axis (equation 1):

$$
\mathrm{ma}_{y}=F_{x f l} \sin \delta_{l}+F_{x f r} \sin \delta_{r}+F_{y f l} \cos \delta_{l}+F_{y f r} \cos \delta_{r}+F_{y r l}+F_{y r r}
$$

Equation of motion of yaw (equation 3):

$$
\begin{aligned}
& \left.J_{z}\right) \&=\left[\left(F_{x f l} \sin \delta_{l}+F_{y f l} \cos \delta_{r}\right)+\left(F_{x f r} \sin \delta_{r}+F_{y f r} \cos \delta_{r}\right)\right] a-\left(F_{y r l}+F_{y r r}\right) b \\
& \left.+\left[F_{y f l} \sin \delta_{l}+F_{x f l} \cos \delta_{l}\right)+\left(F_{x f r} \cos \delta_{r}+F_{y f r} \sin \delta_{r}\right)+\left(F_{x r r}+F_{y r l}\right)\right] \frac{c}{2}
\end{aligned}
$$

Among them, $\mathrm{a}_{\mathrm{x}}$ is the longitudinal acceleration; $\mathrm{a}_{\mathrm{y}}$ is the transverse acceleration; $\mathrm{a}$ is the distance from the centre of mass to the front axle; $m$ is the overall mass of the vehicle; $b$ is the distance from the centre of mass to the rear axle; $\gamma$ is the yaw rate; $J_{z}$ is the moment of inertia of the vehicle body;
$\mathrm{F}_{\mathrm{xfl}}, \mathrm{F}_{\mathrm{xfr}}, \mathrm{F}_{\mathrm{xrl}}$ and $\mathrm{F}_{\mathrm{xrr}}$ are the forces on the x-axis of the four wheels respectively; $\delta, \delta_{l}$, and $\delta_{r}$ are the actual steering angle and the steering angle of the left and right front wheels; $F_{y f l}, F_{y f r}, F_{y r l}$ and $F_{y r r}$ are the forces on the $y$-axis of each wheel; $c$ is the distance between wheels. 


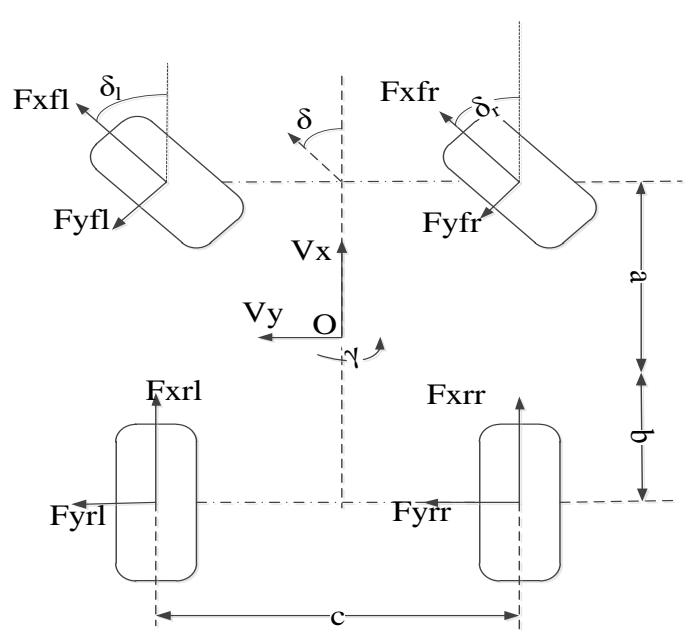

Figure 1: Vehicle dynamics model

Compared with the traditional fuel vehicle, the wheel structure of the four-wheel independent electric vehicle embeds the motor in the wheel, so that the four-wheel independent drive. The expression of vehicle equation is (equation 4):

$$
I_{w} \varpi_{i}=T_{m}-F_{d} r-T_{d}
$$

In the equation, $I_{w}$ is the moment of inertia; $F_{d}$ is the driving force of the tire; $T_{m}$ is the driving torque; $r$ is the wheel radius; $\varpi_{i}$ is the wheel speed; $T_{d}$ is the braking torque. According to the wheel moment balance equation, the force model of the electric wheel as shown in Figure 2 can be drawn.

There are two ways to break the driving wheel of hub motor: electric brake and mechanical brake.

Electric braking is to break through the motor, while mechanical braking is to break through the external brake disc. In order to improve the dynamic performance of the hub motor output, in this study, mechanical braking method is used [9].

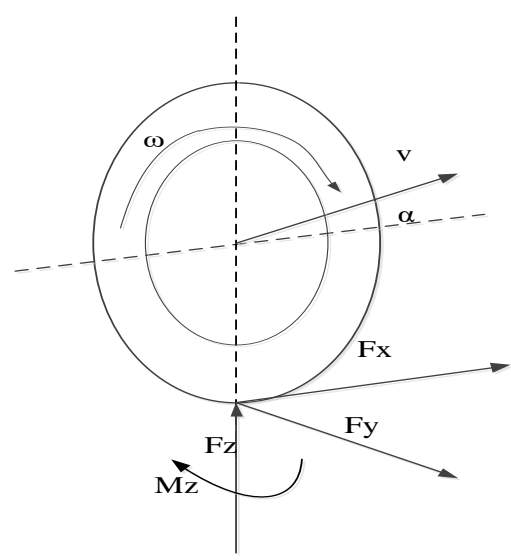

Figure 2: Force model of electric wheel

\subsection{Tire model}

The only way to transmit force and information between vehicle and road surface is tire. At the same time, tire modelling is also an indispensable part of vehicle dynamics analysis, because all vehicle motion patterns are realized through tires. The sideslip characteristic of tire is a very important factor in the analysis of tire mechanical characteristics, which directly affects the handling stability of the vehicle in the process of motion. Therefore, it is necessary to establish an accurate tire model to describe the tire stress. In this study, "Magic Formula" is used to model tire model. Tire force is the core of vehicle dynamics, so it is necessary to analyse the main factors affecting tire force generation, such as tire vertical load, sideslip angle, slip ratio, etc. [10].

(1) Tire vertical load

When the vehicle is driving on the road, the vertical load on the four wheels of the vehicle will change with the change of the vehicle state because of the change of the moving speed and the road condition. The vertical load on each wheel is calculated as follows:

Vertical load of front left wheel $\mathrm{F}_{\mathrm{zfl}}$ (equation 5):

$$
F_{z f l}=m g \frac{b}{2(a+b)}-m a x \frac{h_{g}}{2(a+b)}-m \& \frac{h_{g}}{d} \frac{b}{a+b}
$$

Vertical load of front right wheel $\mathrm{F}_{\mathrm{zfr}}$ (equation 6):

$$
F_{z f l}=m g \frac{b}{2(a+b)}-m \& \frac{h_{g}}{2(a+b)}+m \& \frac{h_{g}}{d} \frac{b}{a+b}
$$

In the equation, $\mathrm{g}$ is the acceleration of gravity; hg is the distance between the vehicle's centre of mass and the ground.

(2) Sideslip angle of tire

The sideslip angle is one of the important factors to characterize the sideslip characteristics of tire. At the same time, the variables that affect the sideslip angle are vehicle running speed and vehicle steering angle.
The expression of vehicle tire sideslip angle is as follows (equation 7):

$$
\alpha_{i}=\delta-\arctan \left(\frac{u_{y i}}{v_{x i}}\right)(i=f l ; f r ; r l ; r r)
$$

In the equation, $\alpha_{i}$ is the sideslip angle of each tire; $\delta$ is the front wheel angle; $\mathrm{u}_{\mathrm{yi}}$ and $\mathrm{v}_{\mathrm{xi}}$ are the 
speed components of four-wheel speeds in the tire plane and vertical tire plane respectively.

The speed component $\mathrm{v}_{\mathrm{xfl}}$ and the sideslip angle

$\alpha_{f l}$ of the front left wheel in the tyre plane are as follows (equation 8-10):

$$
\begin{gathered}
u_{y f l}=v_{y}+a \cdot w_{r} \\
v_{x f l}=v_{x}-\frac{d}{2} \cdot w_{r} \\
\alpha_{f l}=\delta_{f l}-\arctan \left(\frac{u_{y f l}}{v_{x f l}}\right)=\delta_{f l}-\arctan \left(\frac{v_{y}+a \cdot w_{r}}{v_{x}-\frac{d}{2} \cdot w_{r}}\right)
\end{gathered}
$$

(3) Tire slip ratio

There are two movement states of the vehicle tire during the movement: rolling and sliding. Tire slip rate is used to characterize the ratio of tire slip in vehicle motion.

The calculation equation of tire slip rate is (equation 11):

$$
S_{i}=\frac{w_{i} \cdot r-v_{t i}}{v_{t i}}(i=f l ; f r ; r l ; r r)
$$

In the equation, $S_{i}$ is the slip ratio of each tire of the vehicle; $w_{i}$ is the angular speed of each tire of the vehicle; $v_{t i}$ is the longitudinal component of the wheel speed of each tire of the vehicle.

\subsection{PID Control of BLDCM}

In the selection of the control mode of the BLDCM, compared with the more complex fuzzy and neural network control, the PID controller with more extensive industrial control can adjust the speed and torque of the BLDCM very effectively, quickly and simply, so that the four-wheel drive electric vehicle has a more stable output system of the wheel motor [11].

PID control is to adjust the proportion, integral and differential control according to the error.

It is the most widely used control law in the control system. It adjusts the three parameters of proportion, integral and differential gain. PID control schematic diagram is shown in Figure 3.

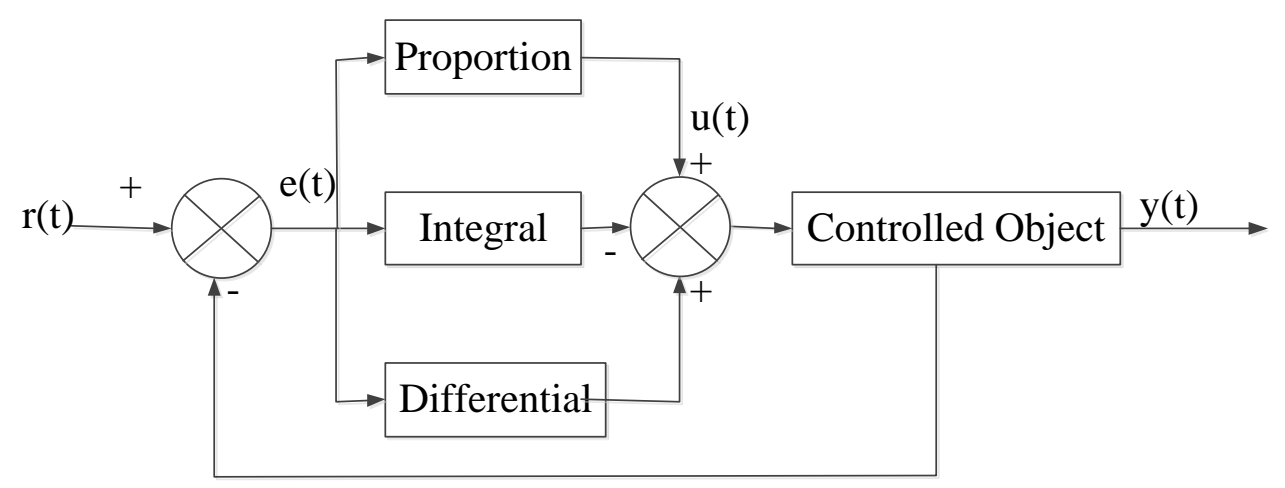

Figure 3: PID control schematic

According to the number of parameters of fuzzy controller, it can be divided into single variable fuzzy controller and multi variable fuzzy controller.

Among them, only one error is selected as the input variable of the single variable fuzzy controller, and the dynamic control performance is poor.

Therefore, the two-dimensional model controller with error e and error variation ec as input can reduce overshoot and system oscillation, so as to improve the stability of the system.

There are six steps in the design of fuzzy controller:

(1) to determine the input and output variables;

(2) to determine the structure of fuzzy controller and the selection of its membership function;

(3) to determine the method of fuzziness and clarity;
(4) to select the range of the input and output variables of fuzzy controller, and to further determine the proportional factor $\mathrm{ku}$ and the quantitative factor ke of the controller. Its parameters are modified online;

(5) finally, to choose a reasonable sampling time, edit the program of its algorithm, online simulation experiment, and compare with the expected effect.

If it is consistent with the expected effect, the design is completed. If it does not meet the expected result, the first step is repeated until it meets the expected effect. In order to achieve the effect of static error, in this system, the form of double input and three output is adopted, and it is combined with PID algorithm to realize the fuzzy controller. When the vehicle stability parameter is not zero, it is necessary to turn on the fuzzy PID controller. 
The controller takes the input as the yaw rate error e and the rate of change of yaw rate error es, and outputs the control parameter yaw moment $\Delta M$ through the fuzzy, fuzzy reasoning and antifuzzy process in the fuzzy controller.

\subsection{Simulation analysis of drive fault tolerance}

The motor faults of the four-wheel drive vehicle are mainly divided into four types: single motor, double motor, three motor and four motor.

When a single motor fails, there are two countermeasures.

The first is to optimize the torque distribution of the other three motors according to the faulttolerant control strategy designed in this study, which is called the three-drive operation simulation.

The second is to artificially set the motor coaxial with the fault motor as the fault motor. At this time, the vehicle becomes the front drive / rear drive (this measure is equivalent to the simultaneous failure of coaxial dual motor).

Then, according to the fault-tolerant control strategy designed in this study, the torque of the front drive / rear drive hub motor is optimally distributed, which is called the front drive / rear drive operation simulation [12].

When two motors on different sides fail, the torque can only be redistributed according to the fault-tolerant control strategy designed in this study. When two motors on the same side fail or three / four motors fail, the vehicle will not be able to continue to drive. The torque distribution of hub motor is essentially a control distribution problem.

Due to the slow development of four-wheel drive electric vehicle in recent years, the research on its control distribution is not enough. However, in the field of flight control system, robot and other industries, control assignment has been widely used.

The traditional control allocation algorithms are: explicit combination method, serial chain method, direct allocation method and so on. With the indepth study of control assignment theory, robust control assignment, nonlinear optimal control assignment and dynamic control assignment appear.

Among them, the nonlinear optimal control allocation algorithms include: piecewise linear programming, quadratic programming, sequential quadratic programming, weighted least squares and so on [13].

Aiming at the complexity of the control system of the four-wheel drive electric vehicle, the sequential quadratic programming (SQP) algorithm developed on the basis of the quadratic programming algorithm is used to distribute the driving torque of the motor.

\section{Results and Discussion}

\subsection{Tire Model modelling and simulation results}

Based on MATLAB / Simulink, the simulation model of vehicle under the joint working condition is established. In order to better characterize the relationship between tire stress characteristics and tire $F_{z}, \alpha, s$, the changes of tire stress under different operating conditions are simulated respectively.

The simulation curve is shown in Figure 4.

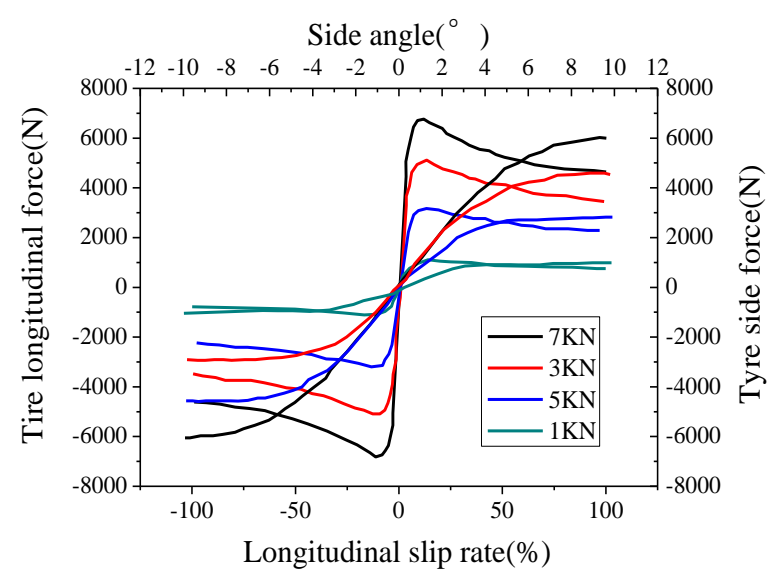

Figure 4: The relationship curve between tire $F_{x}$ and $s$ and relationship between tire $F_{y}$ and $\alpha$

Figure 4 shows the relationship curve between tire $F_{x}$ and $s$ under different $F_{z}$ under pure driving condition. From the curve, it can be seen that the tire under the same load increases with the increase of $\left|F_{x}\right|$. When longitudinal $|s|$ reaches about $10 \%$ or $10 \%, \quad\left|F_{x}\right|$ reaches the maximum value. Subsequently, the tyre $\left|F_{x}\right|$ decreases as $|s|$ increases. Under the same $\mathrm{s}$, the tire $\mathrm{F}_{\mathrm{x}}$ increases with the increase of $\mathrm{F}_{\mathrm{z}}$. Figure 5 shows the relationship curve between tyre $F_{y}$ and $\alpha$ under different loads when the vehicle is in pure turning condition. From the curve, it can be seen that under the same $F_{z}$, the tyre $F_{y}$ increases with the increase of $\alpha$. When $\alpha$ is between $-4^{\circ}$ and $4^{\circ}$, the relationship is linear. In addition, they are nonlinear.

To sum up, in this study, the relationship between the tire force, vertical load, sideslip angle and slip ratio simulated by the tire model is basically consistent with the actual operation of the tire, which shows that the tire model built in this study is correct and credible. 


\subsection{Simulation results of linear operation}

It can be seen from Figure 5 that after motor failure, the characteristic parameters $\mathrm{w}_{\mathrm{r}}$ and $\beta$ of vehicle stable operation using SQP algorithm proposed in this study to reallocate torque are better than those using quadratic planning and average distribution method to follow the expected value.

Among them, the following effect is the worst under the average torque distribution algorithm;

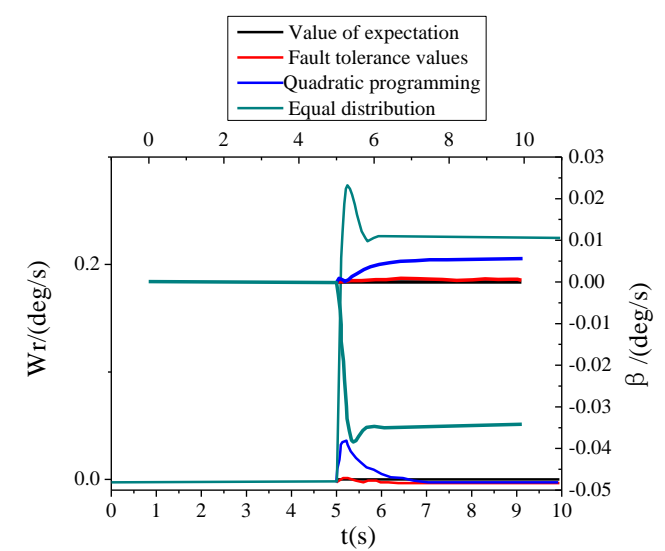

Figure 5: Diagram of vehicle yaw rate and vehicle centroid sideslip angle (straight line)

\subsection{Simulation results of lane changing operation}

Figure 7 shows the $w_{r}$ and $\beta$ changes of the stability operation index of the vehicle during the three-wheel drive operation under the lane changing condition, as well as the torque distribution of the hub motor under the drive fault-tolerant control strategy designed in this study and the lateral displacement change curve which can more intuitively describe the operation state of the vehicle after the motor failure. From Figure 6, it can be seen that the fault-tolerant control strategy and the quadratic planning control strategy designed in this study can well track the expected value of vehicle operation after the vehicle hub motor fails.

However, compared with the two, the control strategy in this study is better than the quadratic planning tracking effect.

The result can be obtained from the running track of the vehicle. When the vehicle runs for a long time in motor failure, the result is more obvious and reduces the safety of drivers and passengers.

After the motor fault, the tracking effect of the average distribution control strategy becomes worse.
If the vehicle operates under this control strategy, an unpredictable danger will occur.

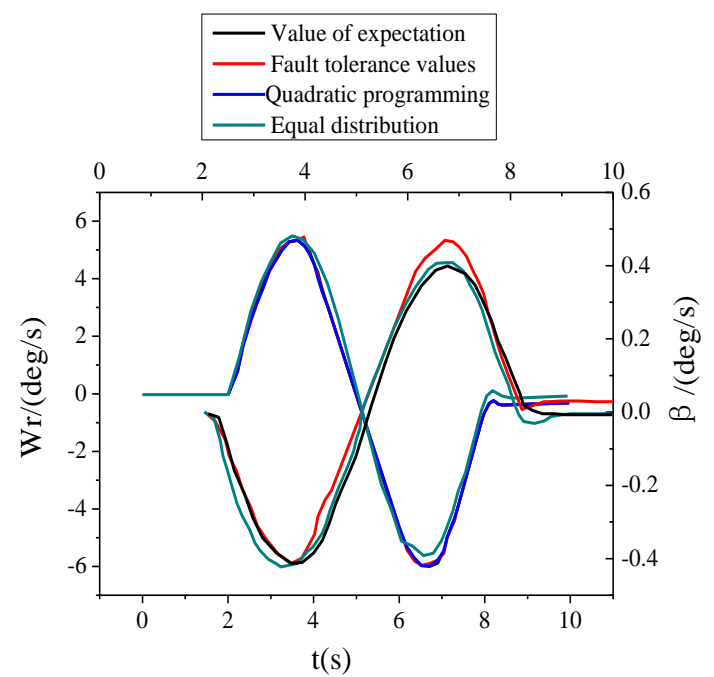

Figure 6: Diagram of vehicle yaw rate and vehicle centroid sideslip (Lane change)

\subsection{Simulation of overtaking operation condition}

Under the condition of overtaking operation, the whole vehicle model is simulated under the same speed, different road adhesion coefficient and the same time, so as to get the running state of the vehicle under different fault-tolerant control algorithms.

The simulation vehicle running speed is $70 \mathrm{~km} /$ $\mathrm{h}$, and the road adhesion coefficient is 0.3 . Through the active fault detection method designed in this study, the change of the running state factors of the right front wheel hub motor and the left rear wheel hub motor is detected in $9 \mathrm{~s}$.

The running results of the vehicle simulation model under overtaking condition, low coefficient of adhesion and simultaneous failure of two motors are shown in Figure 7. It can be seen from the figure that when two diagonal motors fail at the same time, the follow-up effect of $w_{r}$ and $\beta$ fault-tolerant values is better than that of quadratic planning and average distribution, and the vehicle operation is more stable. However, compared with the expected value, there is still a little error, which is caused by the low adhesion coefficient of the road and the deviation of the simulation model.

When the vehicle is running under the condition of low adhesion coefficient and motor failure, if the design of fault-tolerant control strategy is unreasonable, the vehicle will roll over. 


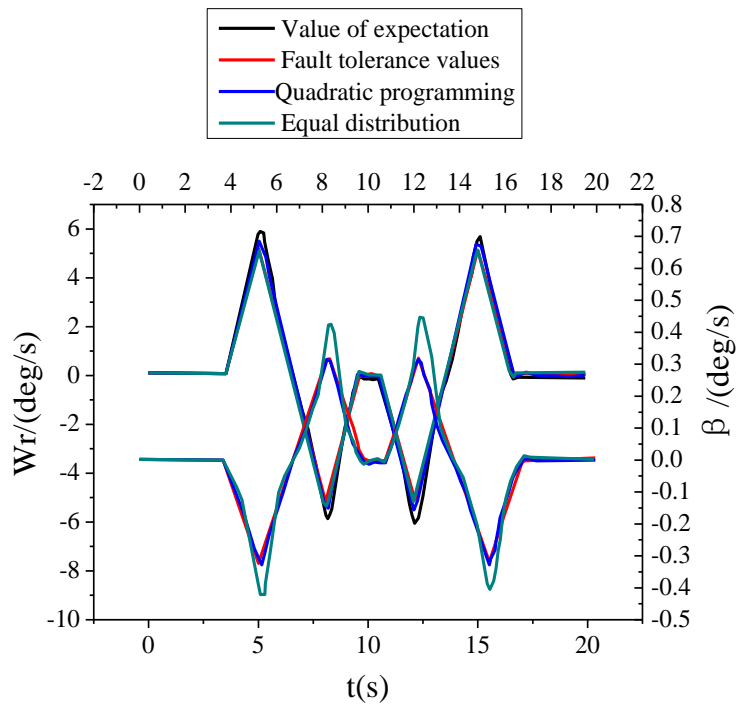

(a) Angular velocity. (b) Angular acceleration.

Figure 7: Diagram of vehicle yaw rate and vehicle centroid sideslip (overtaking)

\section{Discussion}

Compared with the traditional internal combustion vehicle, the four-hub electric vehicle saves a lot of space on the chassis. On the other hand, because there is no complex connecting mechanism, its body is lightweight, which greatly reduces the burden of body weight and saves energy.

However, when the vehicle is turning and driving under complex working conditions, how to coordinate the four-wheel drive speed and make it run stably and safely becomes an important technical problem of the four-wheel independent drive electric vehicle.

In this study, under the designed drive faulttolerant control strategy, a Simulink vehicle control model is built, which is jointly simulated with CarSim. The vehicle simulation model is simulated in three different driving conditions, namely, straight line, lane change, overtaking, at different speeds, at different times, in different sections, and in different positions. Through different simulation results, it is verified that the drive fault-tolerant control strategy designed in this study can effectively control the running stability of the vehicle in different states, so that the vehicle can still run stably in the event of hub motor failure, and improve the safety of drivers and passengers.

Some scholars have studied the fault-tolerant distribution of the driving / braking force of the four-wheel independent drive electric vehicle [14].

Firstly, the four-wheel independent drive electric vehicle is analysed, and the whole vehicle dynamics model is built. Because the four-wheel independent drive electric vehicle system is redundant, the hierarchical design idea is adopted for the faulttolerant control system of hub motor.
The upper layer adopts the robust control method to calculate the force and torque required by the normal driving of the vehicle.

In the lower layer, the real-time adjustment of the distribution matrix is used to redistribute the driving / braking force.

The joint simulation of Simulink and CarSim is used to verify the effectiveness of the fault-tolerant control strategy, which is basically consistent with the results of this study.

The innovation of this study lies in the independent analysis of fault-tolerant control of electric vehicles, which has strong pertinence.

\section{Conclusions}

In this study, the fault-tolerant control strategy, the hub motor system and the motor fault diagnosis of the four-wheel drive electric vehicle have been studied and explored, which provides a certain theoretical and experimental basis for the follow-up study.

Through different simulation results, it is verified that the drive fault-tolerant control strategy designed in this study can effectively control the running stability of the vehicle in different states, so that the vehicle can still run stably in the event of hub motor failure, and improve the safety of drivers and passengers.

In the design of fault-tolerant control strategy, only the influence of yaw rate on vehicle stability is considered. However, in the case of large angle and speed, the influence of centroid sideslip angle on the vehicle is also gradually increasing, so the influence of yaw rate and centroid sideslip angle on the vehicle stability can be comprehensively studied later.

\section{References}

[1] Z. G. Zhao, L. J. Zhou, J. T. Zhang, et al., "Distributed and self-adaptive vehicle speed estimation in the composite braking case for four-wheel drive hybrid electric car "Vehicle System Dynamics, vol. 55, no. 5, pp. 750-773, 2017.

[2] L. Zhai, T. M. Sun, and J. Wang, "Electronic stability control based on motor driving and braking torque distribution for a four in-wheel motor drive electric vehicle", IEEE Transactions on Vehicular Technology, vol. 65, no. 6, pp. 47264739, 2016.

[3] I. Echeverria, F. Arteche, M. Iglesias, et al., "Common mode noise propagation and effects in a four-wheel drive electric vehicle", IEEE Transactions on Electromagnetic Compatibility, vol. 60, no. 1,pp. 132-139, 2017.

[4] L. H. Qiu, L. J. Qian, H. Zomorodi, et al., “Global optimal energy management control strategies for connected four-wheel-drive hybrid electric 
vehicles, "IET Intelligent Transport Systems, vol. 11, no. 5, pp. 264-272, 2017.

[5] A. M.Dizqah, B.Lenzo, A.Sorniotti, et al.,"A fast and parametric torque distribution strategy for four-wheel-drive energy-efficient electric vehicles", IEEE Transactions on Industrial Electronics, vol. 63, no. 7, pp. 4367-4376, 2016.

[6] S. H. Ding, L. Liu, and W. Zheng "Sliding mode direct yaw-moment control design for in-wheel electric vehicles", IEEE Transactions on Industrial Electronics, vol. 64, no. 8, pp. 67526762, 2017.

[7] H. J. Zhou, F.J. Jia, H. H. Jing, et al., "Coordinated longitudinal and lateral motion control for fourwheel independent motor-drive electric vehicle", IEEE transactions on Vehicular Technology, vol. 67, no. 5,pp. 3782-3790, 2018.

[8] D. Zhao, L. Chu, N. Xu, et al., "Development of a Cooperative Braking System for Front-Wheel Drive Electric Vehicles. Energies", vol. 11, no. 2, pp. 378, 2018.

[9] J. S. Hu, Y. F. Wang, H. Fujimoto, et al., "Robust yaw stability control for in-wheel motor electric vehicles", IEEE/ASME Transactions on Mechatronics, vol. 22, no. 3,pp. 1360-1370, 2017.
[10] A.Nasri, B.Gasbaoui, and B. M.Fayssal, "Sliding mode control for four wheels electric vehicle drive", Procedia Technology, vol. 22,pp. 518526, 2016.

[11] D. J. Yin, N. Sun, and J. S. Hu, "A Wheel Slip Control Approach Integrated With Electronic Stability Control for Decentralized Drive Electric Vehicles", IEEE Transactions on Industrial Informatics, vol. 15, no. 4,pp. 22442252, 2019.

[12] J. H. Guo, Y. G. Luo, and K. Q. Li, “An adaptive hierarchical trajectory following control approach of autonomous four-wheel independent drive electric vehicles, "IEEE Transactions on Intelligent Transportation Systems, vol. 19, no. 8,pp. 2482-2492, 2017.

[13] Z. P. Wang, Y.C. Wang, L. Zhang, et al., "Vehicle stability enhancement through hierarchical control for a four-wheel-independentlyactuated electric vehicle", Energies, vol. 10, no. 7, pp. 947, 2017.

[14] L.Zhai, R. F. Hou, T. M. Sun, et al., "Continuous steering stability control based on an energysaving torque distribution algorithm for a four in-wheel-motor independent-drive electric vehicle, "Energies, vol. 11, no. 2,pp. 350, 2018. 